\title{
Contributing role of TNF, IL-10, sTNFR1 and TNF gene polymorphisms in disease severity of leptospirosis
}

\author{
Thilini Nisansala ${ }^{1}$ (D) Manjula Weerasekera ${ }^{1}\left(\right.$ Nilantha Ranasinghe $^{2} \cdot$ Chamil Marasinghe $^{3} \cdot$ Chandika Gamage $^{4}$. \\ Neluka Fernando ${ }^{1}\left[\right.$. Chinthika Gunasekara ${ }^{1}[$
}

Received: 30 November 2019 / Accepted: 14 June 2021 / Published online: 23 June 2021

(c) The Author(s), under exclusive licence to Springer-Verlag GmbH Germany, part of Springer Nature 2021

\begin{abstract}
The immune response is hypothesized as an important factor in the disease outcome of leptospirosis. Exaggerated immune response may promote tissue damage that lead to severe disease outcome. In this study TNF, IL-10, sTNFR1 levels were measured among sixty-two hospitalized leptospirosis confirmed patients in Sri Lanka. Thirty-one serum samples from healthy individuals were obtained as controls. PCR-RFLP method was used to identify TNF gene polymorphisms and to determine their association with TNF expression and disease severity in leptospirosis. TNF $(p=0.0022)$ and IL-10 $(p<0.0001)$ were found to be significantly elevated in leptospirosis patients, while sTNFR1 $(p<0.0001)$ was significantly suppressed. TNF was not significantly elevated in patients with complications while the anti-inflammatory cytokine IL-10 was significantly elevated among patients with complications $(p=0.0011)$ and with mortality $(p=0.0088)$. The ratio of IL-10 to TNF was higher among patients with complications $(p=0.0008)$ and in fatal cases $(p=0.0179)$. No association between TNF gene polymorphisms and TNF expression was detected due to the low frequency of heterozygous and mutated genes present in this study population. Thus the findings of the study show that elevated levels of IL-10 in the acute phase of disease could lead to severe outcomes and a high IL-10/TNF ratio is observed in patients with complications due to leptospirosis.
\end{abstract}

Keywords Leptospirosis $\cdot$ IL-10 $\cdot$ TNF $\cdot$ sTNFR1 $\cdot$ TNF gene polymorphism

\section{Introduction}

The factors contributing to the wide clinical spectrum of leptospirosis ranging from mild flu like symptoms to multiorgan failure and death is still an enigma [1]. It has been suggested that pathogen virulence factors including infecting serovar, extent of leptospiraemia together with host immune response play a major role in disease severity [2]. Besides the direct tissue injury caused by leptospiral components,

Edited by Christian Bogdan.

Chinthika Gunasekara

chinthika@sjp.ac.lk

1 Department of Microbiology, Faculty of Medical Sciences, University of Sri Jayewardenepura, Nugegoda, Sri Lanka

2 Base Hospital, Panadura, Sri Lanka

3 Department of Medicine, Faculty of Medical Sciences, University of Sri Jayewardenepura, Nugegoda, Sri Lanka

4 Department of Microbiology, Faculty of Medicine, University of Peradeniya, Peradeniya, Sri Lanka exaggerated inflammation and host immune suppression have been suggested to contribute to pathogenesis of leptospirosis [3]. During the infection leptospiral structural components such as lipopolysaccharides, haemolysins, outer-membrane proteins and glycoproteins stimulates the innate immune system triggering the inflammatory cascade and release of pro-inflammatory cytokines [4]. Sustained inflammation due to the persistence of the pathogen induces tissue damage leading to production of anti-inflammatory cytokines such as interleukine-10 (IL-10) in the cytokine storm. The cytokine imbalance due to the dynamic production of pro-inflammatory and anti-inflammatory cytokines is an important contributor to pathogenesis determining the disease progression and extent of severity [5].

The pro-inflammatory cytokine tumor necrosis factor alpha (TNF) has been reported to be significantly elevated in patients with leptospirosis [2, 3]. Further higher TNF levels have been observed in patients with severe disease compared to those presenting with mild symptoms highlighting the important role of this cytokine in disease outcome [5, 6]. TNF gene polymorphisms and the expression of soluble 
TNF receptor 1 (sTNFR1) are two important factors which may regulate the role of TNF in disease progression. The role of TNF gene polymorphisms in TNF cytokine expression in leptospirosis has not been studied previously. Gene polymorphisms may contribute to increased or decreased expression of the cytokine and affect the function of TNF [7]. Several polymorphisms have been identified inside the TNF promoter region. $G$ to A polymorphism at -238 and -308 positions are associated with high TNF levels while C to A polymorphism at -863 position is associated with low TNF level [8]. sTNFR1 results from shedding of extracellular domains of membrane bound TNF receptor 1 from cell surface due to proteolytic cleavage. The sTNFR1 can bind to TNF thereby depleting the availability of TNF to bind to cell surface bound TNF receptors. Thus the sTNFR1 in circulation can act as an antagonist neutralizing the function of TNF thereby reducing the inflammatory effect. The findings of several studies suggest that sTNFR1 levels independently could predict mortality or other complications in conditions such as chronic kidney disease (CKD) and inflammatory bowel disease (IBD) among others. In patients with human immunodeficiency virus (HIV) and sepsis the expression of sTNFR1 strongly correlated with clinical stage, progression of disease and thus suggested to be of predictive value [9, 10]. IL-10 which is an anti-inflammatory cytokine inhibits the further release of cytokines by down regulating the Th1 cell response. The balance between TNF and IL-10 could be an important predictor of sequel of disease [6]. The complex role of TNF and IL-10 in disease severity of leptospirosis is still under investigation.

This study aims to identify role of serum TNF, sTNFR1, IL-10 and TNF gene polymorphisms in clinical outcomes of leptospirosis.

\section{Materials and methods}

\section{Study population}

A total of 62 laboratory confirmed leptospirosis patients in medical wards of selected hospitals in Western province of Sri Lanka during January 2017-December 2017 were recruited. Blood samples were collected from all patients on day of admission and day of fever of each patient in relation to day of enrollment for the study was recorded. All the clinical data related to disease presentation, clinical outcome and basic laboratory investigation data were recorded by referring to patients' bed head ticket using an interview based questionnaire. Thirty-one age sex matched healthy individuals were recruited as controls. Blood was collected from patients and healthy controls, serum was separated, aliquoted and stored at $-80{ }^{\circ} \mathrm{C}$ for ELISA assays. A sample of blood was also collected to an ethylene diamine tetra acetic acid (EDTA) tube for deoxyribonucleic acid (DNA) extraction.

Patients with complications were defined as following mentioned. Patients with elevated liver enzymes (more than thrice the upper limit of normal) and elevated serum bilirubin more than $>50 \mu \mathrm{mol} / \mathrm{l}$ were considered as having liver failure. Acute kidney injury (AKI) was defined based on the KDIGO criteria. Chest X-ray appearance consistent with pulmonary haemorrhage was used to identify patients with pulmonary haemorrhage. Clinical features of meningoencephalitis with or without reduced level of consciousness and increased leucocytes in cerebrospinal fluid were determined to having meningitis. Patients with changes of electrocardiogram and echocardiogram patterns were considered as having myocarditis. A patient with more than one complication was considered to be having multi-organ failure. The median day of fever was calculated using the patient history based on the first day of fever reported by the patient. The duration of hospital stay was calculated based on the number of inward days from date of admission.

\section{Laboratory confirmation}

Laboratory confirmation of leptospirosis was performed using a microscopic agglutination test (MAT), real time quantitative PCR (qPCR) and culture isolation of Leptospira.

The MAT was carried out at Medical Research Institute, the reference laboratory for leptospirosis diagnosis in Sri Lanka. The MAT included a panel of 15 reference strains and a MAT titer of $\geq 1: 320$ was considered as positive (cutoff recommended for Sri Lanka by the reference laboratory).

Real-time qPCR based on EvaGreen technology was performed to quantify pathogenic Leptospira spp, using secY IVF (5'-GCG ATT CAG TTT AAT CCT GC-3') and secY IV (5'-GAG TTA GAG CTC AAA TCT AAG-3').

Each experiment was performed using a standard curve and quantity of positive patient samples was determined using it. To establish standard curve, 7-day-old confluent $L$. interrogans serovar Manilae culture grown in liquid $\mathrm{EMJH}$ medium was quantified by measuring the optical density using UV spectrophotometer (UVD-3200) at $420 \mathrm{~nm}$ as described by Hartskeerl re al., [11]. Healthy blood from a volunteer was spiked with quantified culture and a serial dilution of 1,000,000, 100,000, 10,000 and 1,000 Leptospira per ml of whole blood were prepared. DNA extraction was done using QIAamp DNA blood mini kit and then subjected to qPCR assay in triplicates. qPCR was carried out in a total volume of $20 \mu$ containing $5 \mathrm{X}$ qPCR master mix (HOTFIREPol EvaGreen ${ }^{\circledR}$ qPCR Mix Plus, Solis BioDyne, Estonia). Forward and reverse primers were added to a final concentration of $0.4 \mathrm{mM}$ each followed by addition of $10 \mu \mathrm{l}$ of extracted DNA from whole blood using QIAamp DNA blood mini kit (Qiagen GmbH, Germany). qPCR was 
performed on Biorad CFX96 ${ }^{\mathrm{TM}}$ realtime PCR System (BioRad, USA). After an initial denaturation carried out at $95{ }^{\circ} \mathrm{C}$ for 12 min amplification was carried out for 40 cycles comprising of denaturation at $95{ }^{\circ} \mathrm{C}$ for $15 \mathrm{~s}$, annealing at $54{ }^{\circ} \mathrm{C}$ for $30 \mathrm{~s}$, elongation at $72{ }^{\circ} \mathrm{C}$ for $30 \mathrm{~s}$. Melting curve analysis (70-94 ${ }^{\circ} \mathrm{C}$ with reading every $0.5{ }^{\circ} \mathrm{C}$ ) was performed after a cooling step of $30{ }^{\circ} \mathrm{C}$ for $1 \mathrm{~min}$.

$100 \mu \mathrm{l}$ of whole blood was inoculated into semi solid EMJH medium for the culture isolation and detection of Leptospira. All culture media were incubated aerobically at room temperature and kept in dark to protect from light. The cultures were examined weekly for 4 months by placing one drop of the culture on to a microscopic glass slide and observing it by dark-field microscopy at 200 magnification for the presence of spirochetes. Once motile live spirochetes were observed, an aliquot of culture was subjected to DNA extraction using the DNeasy Blood and Tissue kit (Qiagen GmbH, Germany). Amplification of extracted leptospiral DNA in nested $f a B$-PCR was carried out according to the method described previously [12].

\section{Enzyme-linked Immunosorbent assay (ELISA)}

TNF, IL-10 cytokines in serum from the leptospirosis patients and healthy controls were analyzed following the manufacturer's instructions of each ELISA kit (Mabtech, Sweden). Freely available sTNFR1 levels in serum were determined using a commercially available ELISA kit (Elabscience, USA) according to the manufacturer's instructions. Mean absorbance of duplicate samples was calculated and data were analyzed using Graphpad Prism version 7.0 (Graphpad Software Inc) to determine the serum concentrations of each cytokine and sTNFR1.

\section{Detection of TNF gene polymorphisms}

DNA was extracted from $200 \mu \mathrm{l}$ whole blood using QIAamp DNA blood mini kit (Qiagen $\mathrm{GmbH}$, Germany) according to the manufacturer's instructions. To determine TNF gene polymorphisms of leptospirosis patients, PCR and Restriction Fragment Length Polymorphism (RFLP) were performed.

PCR was carried out using the previously published primers which amplify the promoter regions of the TNF gene-238, -308 and -863. Amplification was carried out in a $25 \mu \mathrm{l}$ reaction mixture consisting of $1 \mathrm{X}$ buffer (Sigma Aldrich, USA) with $0.2 \mathrm{mM}$ dNTPs (Promega), $0.2 \mu \mathrm{M}$ of forward and reverse primer, $1.25 \mathrm{U}$ of Taq polymerase (Sigma-Aldrich, USA) and $2 \mu \mathrm{l}(\sim 100 \mathrm{ng})$ of DNA. PCR amplification was performed in a thermal cycler (Flexigene Techne, UK) as described previously [13].

Amplified PCR product was subjected to RFLP using specific restriction enzymes [13]. Both PCR and RFLP products were visualized by agarose gel electrophoresis on a $1.5 \%$ agarose gel and 3\% agarose gel, respectively, under UV transilluminator (VILBER LOURMAT Quantum ST4).

\section{Data analysis}

Data were analyzed using Graphpad Prism version 7.0. As the measured serum TNF, sTNFR1 and IL-10 concentrations did not follow normal distribution, non-parametric statistical tests (Mann-Whitney $U$ test and Kruskal-Wallis test) were used in the statistical analysis. Significant levels were considered at $p<0.05$ and denoted by *.

\section{Results}

\section{Leptospirosis patient characteristics}

The study population consisted of a total of 62 hospitalized leptospirosis confirmed patients and 31 age-sex matched healthy controls. Of the positive cases $79 \%$ (49/62) were positive by real time qPCR (Fig. 1). The median age (IQR) of the patients was $49(36.5-60)$ years and $87 \%$ were males. The median day of fever on admission was 6 days while median duration of hospital stay was 5 days. Elevation of median neutrophil percentage, serum creatinine, serum urea, serum glutamic oxaloacetic transaminase (SGOT), serum glutamate pyruvate transaminase (SGPT) and C-reactive protein (CRP) were observed in this population while

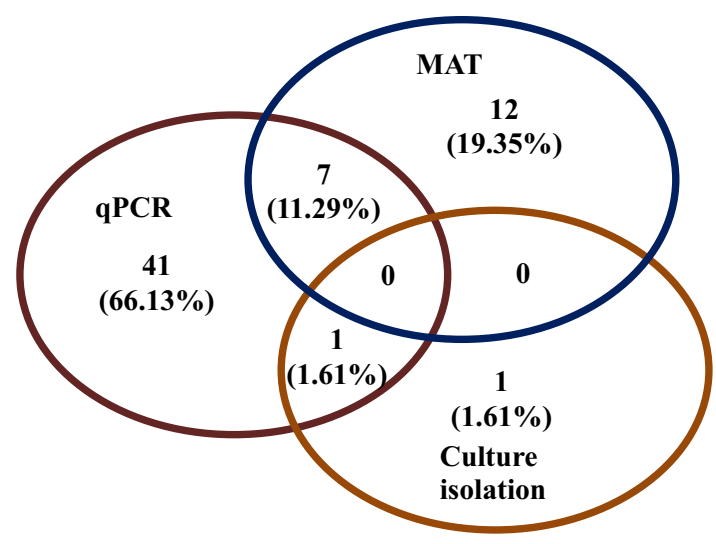

Fig. 1 Confirmation of leptospirosis in 62 positive patients by MAT $(n=19)$, blood culture isolation $(n=2)$ and real time qPCR $(n=49)$. MAT was carried out against a panel of 15 reference Leptospira strains and a titer of $\geq 1: 320$ was considered as confirmatory based on the cut-off given by the national reference laboratory. Culture isolation of Leptospira was done by inoculating whole blood into semi solid EMJH medium. Real-time PCR (Biorad CFX96TM (Bio-Rad, USA)) based on EvaGreen technology was performed to detect pathogenic Leptospira spp, using secYIVF and secYIV. Seven confirmed patients were positive by both MAT and qPCR and one patient was positive by culture isolation and $\mathrm{qPCR}$ 
median haemoglobin and platelet counts were found to be low (Table 1).

Of the 62 leptospirosis patients, a total of $28(45.16 \%)$ patients developed one or more complications and were considered as patients with severe outcomes. The remaining patients without complications were categorized as having mild disease. Among the patients with complications, 19 (67.85\%) had acute kidney injury (AKI), 10 (35.71\%) had liver failure, $10(35.71 \%)$ had pulmonary haemorrhage, 4 (14.28\%) patients had meningitis and $2(7.14 \%)$ patients had myocarditis. Of this group, $13(46.42 \%)$ patients had multi-organ involvement. All most all heamatological and biochemical parameters were significantly varied in leptospirosis patients with complications compared to patients without complications (Table 1). Eight patients were admitted to ICU and 6 patients had fatal outcomes resulting in a case fatality rate of $9.67 \%$.

\section{TNF, IL-10 and sTNFR1 concentrations and severity of disease}

Of the two cytokines evaluated, the expressions of TNF (77.97 vs 35.94, $p=0.0022)$ and IL-10 (37.30 vs 11.86, $p<0.0001)$ levels were significantly elevated in leptospirosis patients compared to healthy controls. The sTNFR1 (18.21 vs $77.59, p<0.0001$ ) was found to be largely suppressed in leptospirosis patients compared to healthy controls (Fig. 2).

The expression of IL-10 ( $p=0.0011)$ was found significantly higher among leptospirosis patients with complications as compared with the patients without complications (Table 2). The median IL-10 concentration of the fatal cases of the study cohort $(n=06)$ was $137.59 \mathrm{pg} / \mathrm{ml}$ (IQR$548.98 \mathrm{pg} / \mathrm{ml}$ ) with a range of $82.45-1167.97 \mathrm{pg} / \mathrm{ml}$. The median IL-10 concentration of nonfatal cases was found to be $36.46 \mathrm{pg} / \mathrm{ml}$ (IQR-81.10 pg/ml) and when levels of IL-10 were compared between fatal cases and survival group there was a significant elevation of IL-10 in fatal group $(p=0.0088)$.

The balance of sTNFR1 to TNF ratio may be important in disease progression as it determines the availability of TNF to bind to cell surface bound TNF receptors. In this study leptospirosis patients had elevated TNF and suppressed sTNFR1. sTNFR1/TNF ratio was significantly higher in healthy controls compared to leptospirosis group (1.227 vs $0.1792, p<0.0000)$ suggesting that in leptospirosis infection there is stronger TNF signaling. When considering the sTNFR1/TNF ratio between leptospirosis patients with complications and without complications, there was no significant difference found between two groups in regard to sTNFR1/TNF ratio ( 0.190 vs $0.114, p=0.6284)$.

It is important to maintain the balance of pro- and antiinflammatory cytokines to prevent immune suppression and thereby resolution of infection. Therefore, in this study, we calculated IL-10 to TNF ratio to seek any association between the balance of pro and anti-inflammatory cytokines and disease severity. The ratio of IL-10 to TNF was significantly higher in leptospirosis group compared to healthy controls $(0.7301$ vs $0.2740, p=0.0019)$. A median of 1.4770 (IQR - 3.4198) was found in the group of patients with leptospirosis complications while 0.3449 (IQR - 0.9240) was found in patients without complications showing a statistically significant elevation in patients with leptospirosis complications $(p=0.0008)$. Similar findings were observable with fatal cases and nonfatal cases and the IL-10 to TNF ratio was significantly elevated in fatal cases than nonfatal cases $(2.4380$ vs $0.4113, p=0.0179)$.

To determine whether there is any difference of evaluated cytokines and sTNFR1 with the day of fever on

Table 1 Biochemical and haematological parameters of leptospirosis confirmed patients $(n=62)$

\begin{tabular}{|c|c|c|c|c|c|}
\hline \multirow{2}{*}{$\begin{array}{l}\text { Haematological /biochemical } \\
\text { parameter (normal range) }\end{array}$} & \multicolumn{2}{|l|}{ Total population $(n=62)$} & \multirow{2}{*}{$\begin{array}{l}\text { Patients with complications } \\
(n=28)\end{array}$} & \multirow{2}{*}{$\begin{array}{l}\text { Patients without com- } \\
\text { plications }(n=34)\end{array}$} & \multirow[t]{2}{*}{$p$ value } \\
\hline & Median (IQR) & Range & & & \\
\hline Haemoglobin (14.0-17.5 g/dl) & $11.60(10.25-12.98)$ & $6.9-15.8$ & $11.06(9.9-12.2)$ & $12.07(10.55-13.2)$ & $0.0353^{*}$ \\
\hline WBC $\left(4000-11,000 / \mathrm{mm}^{3}\right)$ & $10,700(8,070-14,770)$ & $4,750-25,140$ & $12,490(8,830-16,080)$ & $9,210(7,840-13,560)$ & $0.0072 *$ \\
\hline Neutrophils $\left(2000-7000 / \mathrm{mm}^{3}\right)$ & $9,301(5,451)$ & $1,873-20,887$ & $11,050(7,919-13,959)$ & $7,762(6,338-11,054)$ & $0.0015^{*}$ \\
\hline Lymphocytes $\left(1000-3000 / \mathrm{mm}^{3}\right)$ & $1,159(710-2,052)$ & $286-7,080$ & $896(653-1,552)$ & $1,373(782-2,776)$ & $0.0004 *$ \\
\hline Platelet count $\left(150-450 * 10^{3} / \mu \mathrm{l}\right)$ & $91.5(43.0-133.0)$ & $4.0-229.0$ & $80.04(25-118)$ & $108.20(69-146.5)$ & $0.0436^{*}$ \\
\hline $\begin{array}{l}\text { Serum creatinine }(60- \\
120 \mu \mathrm{mol} / \mathrm{l})\end{array}$ & $122.5(96.28-191.6)$ & 55.69-1011 & $313.80(150.10-538.75)$ & $99.15(83.49-131.47)$ & $<0.0001^{*}$ \\
\hline Serum urea $(2.5-6.5 \mathrm{mmol} / \mathrm{l})$ & $11.77(6.8-23.54)$ & $2.9-70.92$ & $20.90(11.55-39.31)$ & $7.20(5.51-12.33)$ & $<0.0001^{*}$ \\
\hline SGOT (10-40 U/l) & $73.25(43.40-104.60)$ & $13.2-852.2$ & $81.75(50.17-113.67)$ & $51.50(35.95-104.1)$ & $0.0112 *$ \\
\hline SGPT (13-31 U/l) & $70.5(41.03-112.7)$ & $18.20-2147$ & $101.30(64.32-161.80)$ & $53.15(36.32-90.22)$ & $0.0045^{*}$ \\
\hline $\mathrm{CRP}<0.5 \mathrm{mg} / \mathrm{l}$ & $180.2(90.73-220.4)$ & $11.30-323.5$ & $190.20(153.30-225.10)$ & $152.80(52.75-218.67)$ & $0.0176^{*}$ \\
\hline Serum bilirubin $(5-21 \mu \mathrm{mol} / \mathrm{l})$ & $19.07(11.1-59.7)$ & $4.5-373.9$ & $55.20(18.26-164.21)$ & $12.26(10.29-19.47)$ & $0.0003^{*}$ \\
\hline
\end{tabular}

${ }^{*} p<0.05$ : Mann-Whitney $U$ test 


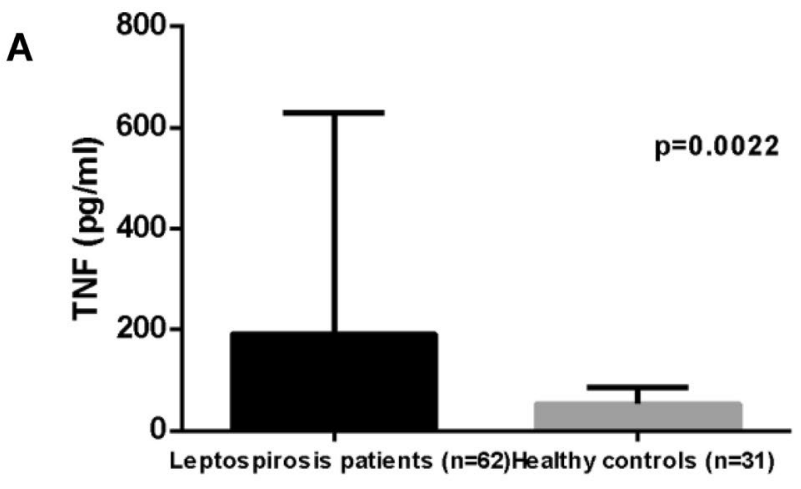

B

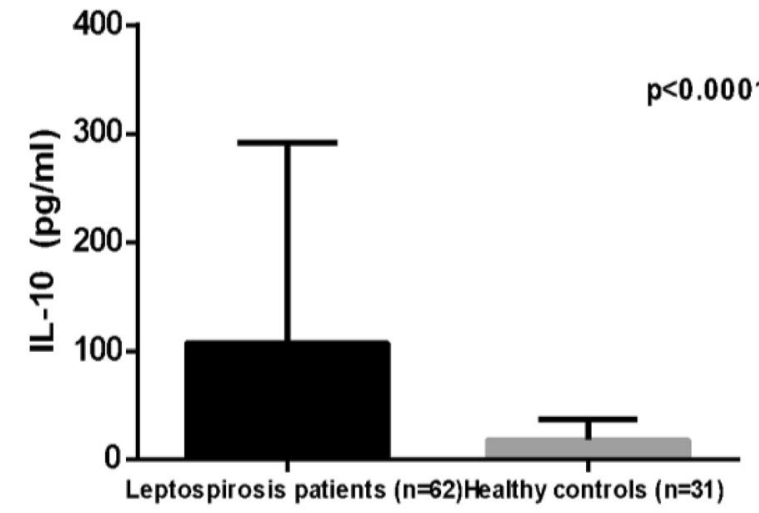

C

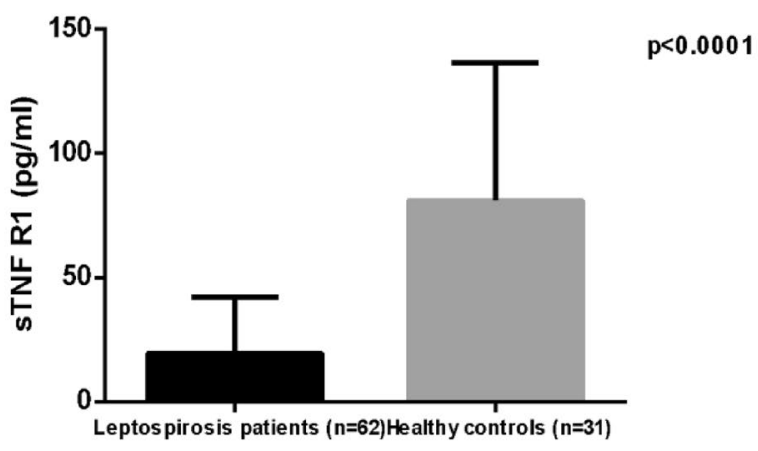

Fig. 2 Comparison of serum TNF, IL-10 and sTNFR1 levels (pg/ $\mathrm{ml})$ in leptospirosis patients $(n=62)$ and healthy controls $(n=31)$. Expression of serum TNF, IL-10 and sTNFR1 in leptospirosis patients and healthy controls were determined by ELISA assays. a Serum TNF expression in patients with leptospirosis and healthy controls; a significantly elevated serum TNF expression $(p=0.0022)$ was observed among leptospirosis patients compared to healthy controls. b Serum IL-10 expression in patients with leptospirosis and healthy controls; a significantly elevated serum IL-10 expression $(p<0.0001)$ was observed in leptospirosis patients compared to healthy controls. c sTNFR1 expression in patients with leptospirosis and healthy controls; a significant suppression of sTNFR1 $(p<0.0001)$ were observed among leptospirosis patients compared to healthy controls

presentation of patient, the patient cohort was divided into two categories as early (fever day of $\leq 6, n=35$ ) and late (fever day of $>6, n=27$ ) presenters. IL-10 expression levels in patients $\leq 6$ days post-onset of illness was significantly elevated than patients with $>6$ days post-onset of illness $(p<0.0001)$. Patients with $\leq 6$ days of illness also had significantly higher ratio of IL-10 to TNF (1.016 vs 0.2833 , $p=0.0038$ ). Results suggest that patients presenting with complications and who presented to the hospital early ( $\leq 6$ days) had significantly higher IL-10 as well as IL-10/ TNF ratio compared to late presenters ( $>6$ days) (Table 3 ).

The bacterial load of patients on admission ranged between $1.21 \times 10^{2}$ and $1.26 \times 10^{6}$ with a median of 3,326 Leptospira/ml (IQR 7,450.83) among patients with a positive PCR of the current study $(n=49)$. The median bacterial load was not statistically different between patients with complications and without complications [median of 2,805.06 (IQR: $8,835.37)$ vs $3,481.66(7,206.11)(p=0.9369)]$. When the expression levels of TNF, IL-10 and sTNFR1 were evaluated with bacterial load of each qPCR positive patient, no correlation was found [Leptospiral load vs TNF $p=0.2215$, IL-10- $p=0.7428$, sTNFR1 $-p=0.7422]$.

\section{TNF gene polymorphisms}

As the TNF gene polymorphisms can affect the production and function of TNF, three TNF gene polymorphisms: TNF -238 , TNF -308 and TNF -863 were investigated among leptospirosis confirmed patients. Majority of patients had the wild type genotype for all three polymorphisms. The wild type genotype was observed in $49(79.0 \%)(\mathrm{TNF}-238 \mathrm{GG})$, $48(77.4 \%)(\mathrm{TNF}-308 \mathrm{GG})$ and 37 (59.7\%) (TNF - 863 $\mathrm{CC})$ patients. Considering the heterozygous genotype among the 62 leptospirosis patients, 8 patients had TNF - $238 \mathrm{GA}$, 14 patients had TNF - 308 GA and 19 patients had TNF - 863 CA polymorphisms. The mutated polymorphism was the rarest genotype reported. Only 5 and 6 patients had TNF - 238 AA and TNF - 863 AA genotypes, respectively, while TNF - 308 AA mutation was not found. There was no significant association between the TNF polymorphisms and TNF cytokine expression in patients as described in Table 4.

\section{Discussion}

The host immune response in leptospirosis is a complex process involving both innate and adaptive immune responses. It is a key factor that determines the outcome of leptospirosis. The innate immune system serves as the first line host defense mechanism which aids in early recognition and elimination of leptospires. This is partially mediated by innate immune cells that trigger inflammatory pathways through the production of cytokines [14]. Studies directed towards the innate immune response in leptospirosis clearly indicate the involvement of $\mathrm{T}$ lymphocytes, neutrophils, macrophages, monocytes and natural killer cells. These cells 
Table 2 Serum concentrations of TNF, IL-10 and sTNFR1 for leptospirosis patients $(n=62)$ and healthy controls $(n=31)$

\begin{tabular}{|c|c|c|c|c|c|c|}
\hline & \multicolumn{3}{|l|}{ Median (range) } & \multicolumn{3}{|l|}{$p$ value } \\
\hline & Healthy controls $(n=31)$ & $\begin{array}{l}\text { Patients without com- } \\
\text { plications }(n=34)\end{array}$ & $\begin{array}{l}\text { Patients with compli- } \\
\text { cations }(n=28)\end{array}$ & $\begin{array}{l}\text { Healthy-with- } \\
\text { out complica- } \\
\text { tions }\end{array}$ & $\begin{array}{l}\text { Healthy-with } \\
\text { complications }\end{array}$ & $\begin{array}{l}\text { Without and } \\
\text { with complica- } \\
\text { tions }\end{array}$ \\
\hline $\mathrm{TNF}(\mathrm{pg} / \mathrm{ml})$ & $35.94(12.31-114.29)$ & $77.97(15.48-3171.42)$ & $78.12(20.06-334.95)$ & $0.0103 *$ & $0.0349 *$ & NS \\
\hline IL-10 (pg/ml) & $11.86(2.01-62.38)$ & $25.38(5.83-195.84)$ & $90.66(6.30-1167.97)$ & $0.0003 *$ & $<0.0001 *$ & $0.0011^{*}$ \\
\hline sTNFR1 (pg/ml) & $77.59(17.47-218.94)$ & $18.56(0-100.87)$ & $\begin{array}{l}17.89 \\
(0-110.52)\end{array}$ & $<0.0001 *$ & $<0.0001 *$ & NS \\
\hline
\end{tabular}

${ }^{*} p<0.05$ : Mann-Whitney $U$ test

NS Not significant

Table 3 Serum concentrations of TNF, IL-10, sTNFR1, IL-10/TNF ratio and sTNFR1/TNF ratio among leptospirosis patients ( $n=62)$ in relation to complications and day of fever

\begin{tabular}{|c|c|c|c|c|c|c|}
\hline & \multicolumn{3}{|c|}{ Patients with complications $(n=28)$} & \multicolumn{3}{|c|}{ Patients without complications $(n=34)$} \\
\hline & \multicolumn{2}{|l|}{ Fever day } & \multirow[t]{2}{*}{$p$ value } & \multicolumn{2}{|l|}{ Fever day } & \multirow[t]{2}{*}{$p$ value } \\
\hline & $\leq 6$ days $(n=15)$ & $>6$ days $(n=13)$ & & $\leq 6$ days $(n=20)$ & $>6$ days $(n=14)$ & \\
\hline IL-10 & 139.0 & 8.970 & $<0.0001 *$ & 33.08 & 18.10 & 0.1101 \\
\hline TNF & 85.27 & 33.47 & 0.1190 & 79.52 & 74.77 & 0.9807 \\
\hline sTNFR1 & 17.80 & 18.44 & 0.8773 & 18.18 & 20.26 & 0.4825 \\
\hline IL-10/TNF ratio & 2.231 & 0.3182 & $0.0050^{*}$ & 0.5563 & 0.2788 & 0.2176 \\
\hline sTNFR1/TNF ratio & 0.1898 & 0.7234 & 0.0760 & 0.07678 & 0.2681 & 0.5893 \\
\hline
\end{tabular}

*p $p$ 0.05: Mann-Whitney $U$ test

Table 4 Concentration of TNF with TNF polymorphisms among patients with leptospirosis

\begin{tabular}{llllll}
\hline Polymorphism & Genotype & $N$ & \multicolumn{2}{l}{ Serum TNF $(\mathrm{pg} / \mathrm{ml})$} & \multirow{2}{*}{$p$ value } \\
\cline { 5 - 6 } & & & Median $(\mathrm{IQR})$ & Range & \\
\hline TNF - 238 & GG (wild type) & 49 & $72.7(32.3-107.0)$ & $15.5-1171.0$ & 0.1088 \\
& GA (heterozygous) & 08 & $94.5(82.9-198.2)$ & $73.2-277.6$ & \\
& AA (mutated) & 05 & $79.4(53.6-1794.3)$ & $39.1-3171.4$ & \\
TNF -308 & GG (wild type) & 48 & $79.4(41.2-135.2)$ & $15.5-1171.0$ & 0.9482 \\
& GA (heterozygous) & 14 & 73.57 & $16.5-3171.4$ & \\
& AA (mutated) & 00 & - & & \\
TNF -863 & CC (wild type) & 37 & $81.0(58.0-139.6)$ & $15.5-3171.4$ & 0.5544 \\
& CA (heterozygous) & 19 & $76.2(31.4-207.4)$ & $16.5-417.1$ & \\
& AA (mutated) & 06 & $69.8(23.5-124.5)$ & $20.0-220.8$ & \\
\hline
\end{tabular}

$* p<0.05:$ Kruskal-Wallis test aid in the activation of antimicrobial responses and production of pro/anti-inflammatory cytokines $[2,15]$. Thus the observed IL-10 elevation in patients further supports the possibility of immune suppression due to leptospirosis.

TNF is a pro-inflammatory cytokine which has been largely investigated in leptospirosis patients [3, 16]. In this study TNF expression was high in the patient group compared to the healthy controls. Elevation of TNF in leptospirosis patients have been reported in several studies [2, 17].
TNF production due to pathogenic Leptospira is stimulated through the toll like receptor dependent mechanisms. The pro-inflammatory function of TNF is exerted through binding to the TNFR1 which is expressed in almost all cells of the body [18, 19]. Cell surface expressed TNFR1 is cleaved and released as a soluble form, which is known to scavenge TNF in the blood thus making TNF unavailable for biological function. 
In this study, the comparison of TNF expression between patients with and without complications, revealed no significant difference between these two groups. Several other studies have reported controversial results of TNF expression in leptospirosis. A study by Wagenaar et al. reported low or un-detectable TNF concentrations among leptospirosis patients, while Reis et al. reported elevated expression of TNF in severe leptospirosis [5, 20]. However, another study reported significantly low levels of TNF among fatal cases compared to nonfatal cases [6]. According to Papa et al., TNF was significantly lower in the cases with pulmonary involvement [4].

A significant decrease in STNFR1 was observed in the patients compared to the healthy controls in this study. sTNFR1 binds to TNF thereby acting as a TNF inhibitor. In the absence of sTNFR1, TNF can exert pro-inflammatory signaling which can subsequently lead to severe consequences. Different stimuli, including TNF itself enhance the release of sTNFR1 into the circulation. Thus higher TNF produced in leptospirosis patients compared to healthy controls may enhance the proteolytic cleavage of TNFR1 resulting in increased shedding of sTNFR1 into circulation and facilitating its binding with the cytokine. However, low levels of sTNFR1 among leptospirosis patients in general could be due to binding of sTNFR1 to TNF and thus interfering with its detection.

This is the first genetic study carried to determine the association of TNF gene polymorphisms with TNF expression level among patients with leptospirosis in Sri Lanka to our knowledge. Majority of the patients in this study expressed the wild type genotype, thus it was not possible to determine an association between gene polymorphisms and TNF production. In 2004, a study by Lingappa et al., found no significant association between TNF 308 gene polymorphism and leptospirosis [21]. In studies investigating the TNF gene polymorphisms among a group of dyspeptic patients and Dengue patients from Sri Lanka, a low frequency of heterozygous and mutated genotype was reported suggesting a predominance of the wild type genotype among Sri Lankans [13, 22]. A similar observation of a low frequent of heterozygous and mutated TNF 238, 308 and 863 was noted by Mishra et al., among an Indian population during the course of pandemic Influenza [23].

IL-10, an anti-inflammatory cytokine was elevated among leptospirosis patients when compared with healthy controls. The balance between pro- and anti-inflammatory cytokines has been recognized as an important factor in the progression of disease [3]. Reis et al. demonstrated that IL-10 was an independent predictor of death in leptospirosis [5]. This is also reflected in the current study where patients whose outcome was fatal had high IL-10 expression. Similarly patients with leptospirosis disease complications also had significantly high IL-10 expression compared to those without complications. Reis et al. suggested that over production of IL-10 shifts the immune response from Th1 to a Th2 dominated response [5]. IL-10 mediated immunosuppression down regulates the monocyte derived TNF expression. Besides, IL-10 derived immune suppression with other several factors can lead to multi-organ failure and death. Association of IL-10 with death and disease severity has been reported by several other groups $[5,6]$. However, another research group stated that the higher levels of IL-10 were found to be associated with better prognosis in leptospirosis [24]. Moreover, patients presenting in early stage of the disease had significantly elevated IL-10 expression in this study whereas Papa et al., found elevated IL-10 levels during 6-10 days post-onset of illness [4].

Several groups have investigated the importance of IL-10/TNF ratio in correlation with the disease severity as it depicts the balance between these important pro- and anti-inflammatory cytokines. However, the results reported by several research groups are conflicting. Current study reported significantly elevated IL-10/TNF ratio among leptospirosis patients compared to healthy individuals, patients with leptospirosis complications and fatal cases. However, studies by Rizvi et al., and Tajiki et al., found a high IL-10/ TNF ratio in patients with better prognosis which is in contrast to our findings [24, 25]. Kyriakidis et al., reported a significantly higher IL-10/TNF ratio among fatal cases, thus suggesting IL-10/TNF ratio as a useful biomarker of prognosis in leptospirosis in their study [6]. Further in the current study, a significantly high ratio of IL-10/TNF was seen in the early phase of disease ( $\leq 6$ days) as opposed to late phase ( $>6$ days) of fever. This finding suggests that the ratio of IL-10/TNF may be a useful early predictor of disease prognosis which is an important aspect for further consideration.

Although there are conflicting reports regarding the associations of cytokine expression and outcome of leptospirosis by several groups, it is possible that the sampling and methodology of these studies could contribute to the contradictory results seen. These observed differences in the cytokine expression of IL-10 and TNF could be attributed to several factors including the time point of sample collection in relation to the onset of the symptoms, selection of the laboratory confirmatory tests (e.g.: PCR, MAT, ELISA, etc.) and use of different commercially available cytokine assays and techniques (pre-coated or uncoated ELISA techniques, multiarray assays, inflammatory colorimetric bead array etc.). The cytokine half-life during long term storage is an important influencing factor that should be carefully considered to avoid discrepancies in cytokine measurements. In addition, the selection of sample size could influence the findings. Patient recruitment of different studies may vary according to their own specific healthcare settings and recruitment criteria such as inward/ICU patients or outdoor patients [26]. Subsequently, the lack of clear categorization criteria 
to define mild/moderate or severe disease in leptospirosis is another factor that should be considered when interpreting findings of each study. Recruitment of inward patients was one limitation of this study. In the local setting patients seeking hospital care are clinically symptomatic patients requiring hospital admission and thus this will introduce a bias in sampling. This may result in insufficient inclusion of patients with mild symptoms who do not seek hospital care. It is well known that there are $>250$ pathogenic serovars and thus the circulating serovars of Leptospira is known to show geographical variation. The pathogen and host genetic factors, could thus affect the host immune response including cytokine expression. Fernando et al., revealed a probable down regulation of pro-inflammatory cytokines by administrating ceftriaxone in leptospirosis patients [27]. This suggests that antibiotic treatment is also a determining factor which influences the levels of cytokine expression.

\section{Conclusion}

Thus the findings of the study show that elevated levels of IL-10 in the acute phase of disease could lead to severe outcomes and a high IL-10/TNF ratio is observed in patients with complications due to leptospirosis. This finding could be further validated in a larger study to determine the clinical application in patient management.

Acknowledgements We wish to acknowledge all patients, staff of relevant hospitals and the members of the Department of Microbiology, Faculty of Medical Sciences, University of Sri Jayewardenepura, Sri Lanka.

Author contributions TN involved in sample and data collection, laboratory work, data analysis and interpretation and writing the manuscript. MW designed the study, involved in proposal writing, data analysis and writing the manuscript. NR and CM selected and recruited the patients for the study and involved in drafting the article. CDG involved in laboratory diagnosis and writing the manuscript. NF involved in proposal writing, research question, data analysis and writing the manuscript. CG involved in proposal writing, designed the study, research question, data analysis, writing the manuscript. All authors read and approved the final manuscript.

Funding This work was supported by the Grants awarded by University of Sri Jayewardenepura, Sri Lanka. [ASP/01/RE/MED/2015/37, ASP/01/RE/MED/2016/48 and ASP/01/RE/MED/2017/29].

\section{Declarations}

Conflict of interest We declare that we have no conflict of interest.

Ethics approval Ethical clearance for the study was obtained from the Ethics Review Committee (ERC) of the University of Sri Jayewardenepura, Sri Lanka (ERC Application No. 02/17) and from the regional director of health services and the respective hospitals.
Informed consent Informed written consent was obtained from the patient prior to enrollment.

\section{References}

1. Haake DA, Levett PN (2015) Leptospirosis in humans. Leptospira and leptospirosis. Springer, pp 65-97

2. Bandara K, Gunasekara C, Weerasekera M, Marasinghe C, Ranasinghe N, Fernando N (2018) Do the Th17 cells play a role in the pathogenesis of leptospirosis? Can J Infect Dis Med Microbiol. https://doi.org/10.1155/2018/9704532

3. Cagliero J, Villanueva SYA, Matsui M (2018) Leptospira pathophysiology: into the storm of cytokines. Front Cell Infect Microbiol 8:204

4. Papa A, Kotrotsiou T (2015) Cytokines in human leptospirosis. Trans R Soc Trop Med Hyg 109(12):749-754

5. Reis EA, Hagan JE, Ribeiro GS, Teixeira-Carvalho A, MartinsFilho OA, Montgomery RR et al. Cytokine response signatures in disease progression and development of severe clinical outcomes for leptospirosis. PLoS Negl Trop Dis. 2013;7(9):e2457.

6. Kyriakidis I, Samara P, Papa A (2011) Serum TNF- $\alpha$, sTNFR1, IL-6, IL-8 and IL-10 levels in Weil's syndrome. Cytokine 54(2):117-120

7. Qidwai T, Khan F (2011) Tumour necrosis factor gene polymorphism and disease prevalence. Scand J Immunol 74(6):522-547

8. Fargion S, Valenti L, Dongiovanni P, Fracanzani AL. TNF $\alpha$ Promoter Polymorphisms. Tumor Necrosis Factor. Springer; 2004. p. 47-58.

9. Spoettl T, Hausmann M, Klebl F, Dirmeier A, Klump B, Hoffmann J et al (2007) Serum soluble TNF receptor I and II levels correlate with disease activity in IBD patients. Inflamm Bowel Dis 13(6):727-732

10. Moreira JM, da Silva AN, Vieira ÉLM, Teixeira AL, Kummer AM, e Silva ACS. Soluble tumor necrosis factor receptors are associated with severity of kidney dysfunction in pediatric chronic kidney disease. Pediatric Nephrology. 2019;34(2):349-52.

11. Hartskeerl R, Smits H, Korver H, Goris M, Terpstra W, Fernández C (2001) International course on laboratory methods for the diagnosis of leptospirosis. Royal Tropical Institute Department of Biomedical Research, Netherlands

12. Nisansala G, Muthusinghe D, Gunasekara T, Weerasekera M, Fernando S, Ranasinghe $\mathrm{K}$ et al (2018) Isolation and characterization of Leptospira interrogans from two patients with leptospirosis in Western Province, Sri Lanka. J Med Microbiol 67(9):1249-1252

13. Arachchi PS, Weerasekera MM, Senevirathna B, Weerasekera D, Fernando N, Gunasekara CP (2018) Association of tumor necrosis factor alpha gene polymorphisms with Helicobacter pylori infection in dyspeptic patients in Sri Lanka. Microbiol Immunol 62(7):429-435

14. Vernel-Pauillac F, Werts C (2018) Recent findings related to immune responses against leptospirosis and novel strategies to prevent infection. Microbes Infect 20(9-10):578-588

15. Lindow JC, Tsay AJ, Montgomery RR, Reis EA, Wunder EA Jr, Araújo G et al (2019) Elevated activation of neutrophil toll-like receptors in patients with acute severe leptospirosis: an observational study. Am J Trop Med Hyg 101(3):585-589

16. Estavoyer J, Racadot E, Couetdic G, Leroy J, Grosperrin L (1991) Tumor necrosis factor in patients with leptospirosis. Rev Infect Dis 13(6): 1245

17. Chirathaworn C, Supputtamongkol Y, Lertmaharit S, Poovorawan Y (2016) Cytokine levels as biomarkers for leptospirosis patients. Cytokine 85:80-82 
18. Goris MG, Wagenaar JF, Hartskeerl RA, van Gorp EC, Schuller S, Monahan AM et al (2011) Potent innate immune response to pathogenic leptospira in human whole blood. PLoS ONE 6(3):e18279

19. Yang S, Wang J, Brand DD, Zheng SG (2018) Role of TNF-TNF receptor 2 signal in regulatory $\mathrm{T}$ cells and its therapeutic implications. Front Immunol. https://doi.org/10.3389/fimmu.2018.00784

20. Wagenaar JF, Gasem MH, Goris MG, Leeflang M, Hartskeerl RA, van der Poll T et al (2009) Soluble ST2 levels are associated with bleeding in patients with severe Leptospirosis. PLoS Neglect Trop Dis. 3(6):e453

21. Lingappa J, Kuffner T, Tappero J, Whitworth W, Mize A, Kaiser $R$ et al (2004) HLA-DQ6 and ingestion of contaminated water: possible gene-environment interaction in an outbreak of Leptospirosis. Genes Immun 5(3):197

22. Fernando AN, Malavige GN, Perera KLN, Premawansa S, Ogg GS, De Silva AD (2015) Polymorphisms of transporter associated with antigen presentation, tumor necrosis factor- $\alpha$ and interleukin-10 and their implications for protection and susceptibility to severe forms of dengue fever in patients in Sri Lanka. J Glob Infect Dis 7(4):157

23. Saud B, Sharma M, Mishra B, Sarkar S, Ratho R (2016) TNFalpha gene polymorphism in Indian population during the course of pandemic influenza a H1N1 infection: a pilot study. MOJ Immunol 3(5):00100
24. Rizvi M, Azam M, Sultan A, Khan F, Shukla I, Malik A et al (2014) Role of IL-8, IL-10 and TNF- $\alpha$ level in pathogenesis of leptospiral acute hepatitis syndrome. Ann Pathol Lab Med 1(02):A10-A17

25. Tajiki MH, Satie AN, Salomão R (1997) The ratio of plasma levels of IL-10/TNF-alpha and its relationship to disease severity and survival in patients with leptospirosis. Braz J Infect Dis 1(3):138-141

26. Senavirathna I, Rathish D, Agampodi S (2020) Cytokine response in human leptospirosis with different clinical outcomes: a systematic review. BMC Infect Dis 20:1-8

27. Fernando N, de Silva R, Handunnetti SM, Karunanayake L, De Silva NL, de Silva HJ et al (2018) Effect of antimicrobial agents on inflammatory cytokines in acute leptospirosis. Antimicrob Agents Chemother. https://doi.org/10.1128/AAC.02312-17

Publisher's Note Springer Nature remains neutral with regard to jurisdictional claims in published maps and institutional affiliations. 